\title{
Localization of Trochlear Spine \& Fovea in Human Skulls
}

${ }^{1}$ Iram Atta, ${ }^{1}$ Maria Nouman, ${ }^{2}$ Raafea Tafweez

${ }^{1}$ Department of Anatomy, King Edward Medical University, Lahore

${ }^{2}$ Department of Anatomy and Histology, King Edward Medical University, Lahore

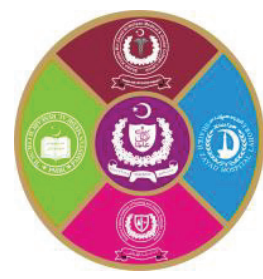

\begin{abstract}
Introduction: Many otolaryngologist and the orbital surgeons have been devoted to finding a better way to achieve optimal outcome in fields of the paranasal sinuses and surgery on medial orbital wall. This could be achieved when the surgeon gives sufficient consideration to orbital anatomy. The cartilaginous pulley of superior oblique muscle is vulnerable during the operations on frontoethmoidal sinus. This pulley passes through an anatomical landmark called trochlear fovea and spine Aims \& Objectives: To describe proper location of trochlear fovea and spine by using the measurements of frontal and sagittal plane. Place and duration of study: The study was conducted at King Edward Medical University, Lahore in 2020 on 61 dry skulls. Material \& Methods: Four measurements were taken in frontal plane using two lines passing at right angle through supra orbital notch and fronto zygomatic suture while in sagittal plane the distance of fovea/spine was measured from orbital margin anteriorly and optic canal posteriorly. Results: The distance of fovea and spine from the lines along supra orbital notch and fronto zygomatic suture was $7.22 \pm 0.93 \mathrm{~mm}$ and $6.14 \pm 0.83 \mathrm{~mm}$ respectively. It was $3.77 \pm 0.73 \mathrm{~mm}$ behind the margin and $38.22 \pm 2.98 \mathrm{~mm}$ in front of optic canal. Conclusion: To prevent unwanted injury to superior oblique pulley surgeons should know the topographic location and variation of fovea and spine so that the postoperative diplopia could be prevented while approaching the paranasal sinuses and surgery on medial orbital wall.
\end{abstract}

ABSTRACT

Key words: Trochlear spine, Trochlear fovea, Orbital cavity

\section{INTRODUCTION}

$\mathrm{W}$ ith the advancement of surgical techniques in the field of ophthalmology, more studies on the relations of various orbital structures is needed so that the postoperative complications of these procedures could be avoided. ${ }^{1}$ The eyeball is moved by the extra ocular muscles. The strap shaped four recti arise from the common tendinous ring located at the orbital apex. As these muscles pass forward from the apex they broaden out and form cone of muscles around the eye. ${ }^{2}$ The orientation of recti is in such a way that lateral rectus is parallel to the lateral wall of the orbit, medial rectus is along a sagittal plane, the superior and inferior recti following the orbital axis roughly forms an angle of $23^{\circ}$ with the medial and lateral walls of the orbit. ${ }^{3}$ On the other hand, the two oblique muscles approach the eyeball in a front to back and medial to lateral direction. Inferior oblique, a thin, narrow muscle arises from floor of the orbit and is inserted into the lateral part of the sclera behind the equator of the eyeball. Whereas the superior oblique, a fusiform muscle arises from the body of the sphenoid superomedial to the optic canal and runs forwards to end in a round tendon that passes through a fibrocartilaginous loop or pulley, the trochlea, which attached to the trochlear fovea of the frontal bone and the tendon then gets attached to the sclera in the superolateral part of the posterior quadrant behind the equator. ${ }^{4}$ The trochlear fovea is a 3-5 $\mathrm{mm}$ shallow depression on the frontal bone at the anteromedial aspect of the orbital roof. Bony spicula called as trochlear spine sometimes arises from this fovea. Their location is at the superomedial angle of orbit being 4-5 $\mathrm{mm}$ behind the orbital opening ${ }^{5}$. Localization of trochlear fovea and spine is of great importance for the ophthalmologist while dealing with the frontal sinus surgeries as many cases of post-operative diplopia have been reported due to injury to trochlea during the surgical procedure. ${ }^{6,7}$ Moreover repair of fractures at the orbital roof could also result in disruption of the trochlea leading to visual disparity. ${ }^{8}$ Therefore, to provide ophthalmologist with some useful references on measurements of human dry skulls which were used to document 
incidence and variation in the anatomical location of trochlear fovea and spine in Pakistani population.

\section{MATERIAL AND METHODS}

The study was conducted on 61 dry adult human skulls stored in the Department of Anatomy, King Edward Medical University, Lahore, Pakistan. Overall 122 orbits were observed. Out of these the data could be collected from 120 orbits as 2 were spoiled. All the skulls were examined to determine the gender, incidence and exact location of trochlear fovea or spine. The sex of the skull was determined by the morphological discriminant method. ${ }^{9}$ In order to locate the trochlear fovea or spine two lines were drawn in the frontal plane from the supraorbital notch and fronto zygomatic suture respectively. These two significant anatomical landmarks were used to measure the interval of trochlear spine and fovea from the roof, medial wall, supraorbital notch and fronto zygomatic suture. A vertical line was drawn from the supraorbital notch (L1) and a horizontal line was drawn through fronto zygomatic suture in the frontal plane (L2). (L3) and (L4) were drawn through the trochlear fovea and spine base transversely and vertically respectively. Distances measured between the base of the spine and center of the fovea and L1 and L2 were recorded as D1 and D3 respectively (Fig-1), Gap between L3 and the orbital roof measured along L1 is shown as D2. Interval between L4 and the medial wall of the orbit measured along L2 is represented as D4 (Fig-2). Further two measurements were taken in the sagittal plane to localize the trochlear fovea and spine. D5 was measured from the anterior orbital opening and the base of the trochlear spine and center of the fovea so D6 was recorded from the anterior margin of the optic canal and the base of the trochlear spine and center of the fovea (Fig-3). All the readings were recorded with the Vernier calipers and were taken in millimeters.

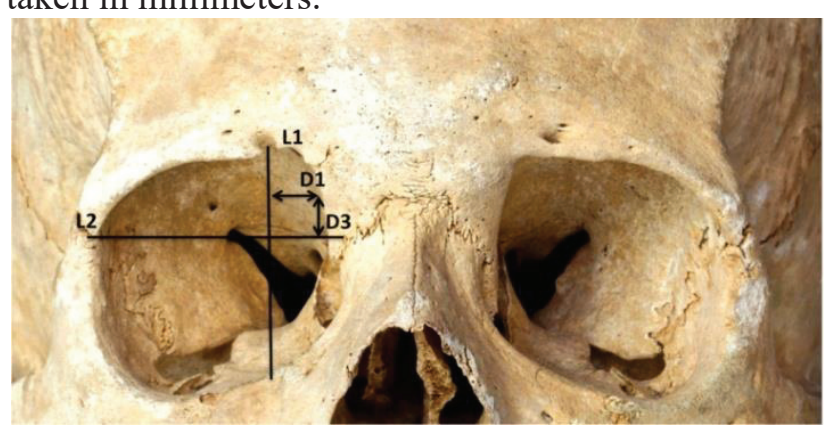

Fig-1: (L1) and (L2) are shown passing through the supraorbital notch and fronto zygomatic suture respectively. (D1) indicates the interspace between trochlear fovea/spine and (L1) while (D3) indicates the interspace from (L2).

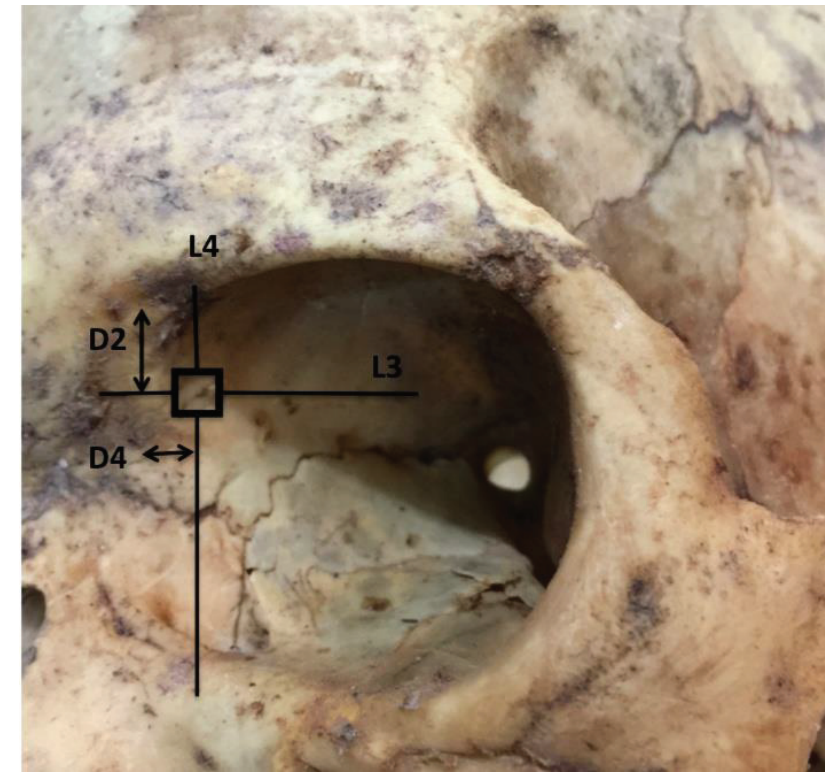

Fig-2: (L3) and (L4) are drawn perpendicular to each other through the trochlear fovea/spine. Along L1, D2 was recorded between orbital roof and L3. Moreover, D4 was taken along L2 between the medial wall of the orbit and L4.

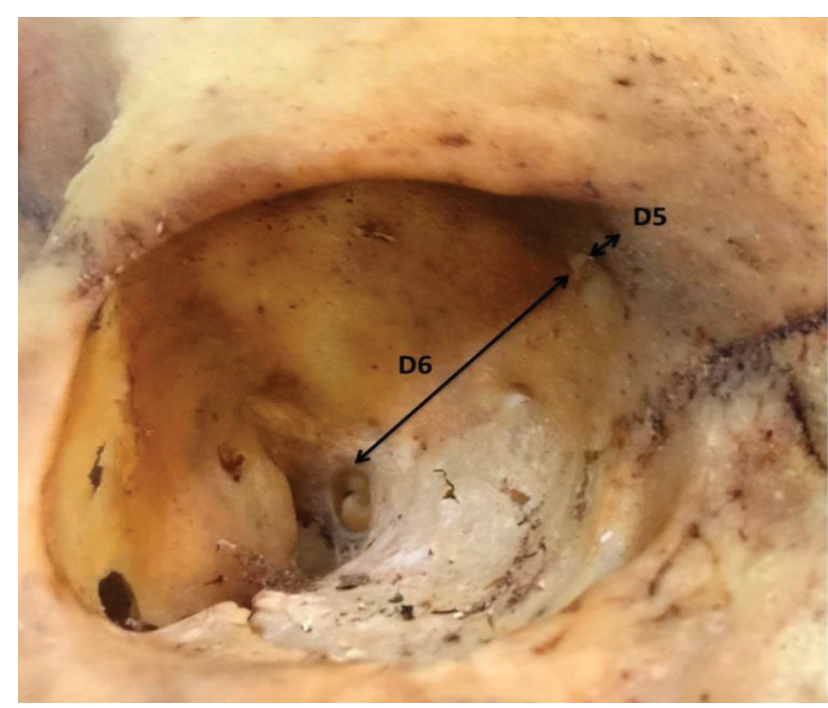

Fig-3: D5 is shown along the sagittal plane from the anterior orbital opening and trochlear fovea or spine. D6 represents the distance of spine or fovea from the optic canal.

\section{Statistical analysis:}

Data was entered and analyzed by using SPSS-21-. Student t-test was applied to authenticate the difference between male and female position of trochlear fovea and spine. Significance between the incidence of the trochlear fovea and spine in male and female skulls was tested by Z-test. $\mathrm{P}<0.05$ was taken as significant. 


\section{RESULTS}

120 orbits were studied in which trochlear fovea was present in 101 orbits and trochlear spine was observed in 20 orbits.

\begin{tabular}{|c|c|c|c|c|c|}
\hline \multicolumn{3}{|c|}{ Trochlear fovea } & \multicolumn{3}{c|}{ Trochlear spine } \\
\hline Side & Number & $\%$ & Side & Number & $\%$ \\
\hline Right & 55 & 54.45 & Right & 15 & 75 \\
\hline Left & 46 & 45.54 & Left & 5 & 25 \\
\hline Total & 101 & 84.17 & Total & 20 & 16.67 \\
\hline
\end{tabular}

Table-1: Incidence of trochlear fovea and spine

The incidence of trochlear fovea was found to be $84.17 \%$ with more prevalence on right side. Whereas the incidence of spine was $16.67 \%$ with the percentage on right being more than on left. Moreover t-test showed that there was no sex related preference of trochlear fovea and spine as the frequency of fovea in male $(84.2 \%)$ and female $(80 \%)$ showed no statistical difference. Similarly, percentage of trochlear spine in male $(16.7 \%)$ and female $(19.8 \%)$ did not show any significant difference statistically.

\begin{tabular}{|c|c|c|c|}
\hline $\begin{array}{c}\text { Distance } \\
\text { measured }\end{array}$ & $\begin{array}{c}\text { Mean } \\
(\mathbf{m m})\end{array}$ & $\begin{array}{c}\text { Maximum } \\
\mathbf{( m m})\end{array}$ & $\begin{array}{c}\text { Minimum } \\
\mathbf{( m m})\end{array}$ \\
\hline D1 & $7.22 \pm 0.93$ & 8.64 & 5.35 \\
\hline D2 & $4.86 \pm 0.93$ & 6.98 & 3.04 \\
\hline D3 & $6.14 \pm 0.83$ & 8.13 & 4.43 \\
\hline D4 & $0.98 \pm 0.48$ & 1.92 & 0.10 \\
\hline
\end{tabular}

Table-2: Measurements of trochlear spine and fovea in frontal plane

Where D1 is the distance measured from fovea/ spine till supraorbital notch (L1).

D2 is the distance measured from fovea/ spine till orbital roof along L3.

D3 is the distance measured from fovea/ spine till fronto zygomatic suture (L2).

D4 is the distance measured from fovea/ spine till medial wall of orbit along L4.

On applying t- test values of D1 and D4 showed no statistical difference in male and female whereas D2 and D3 were significantly different in male and female as depicted in Table-3

\begin{tabular}{|c|c|c|c|c|}
\hline & $\begin{array}{c}\text { D1 mean } \\
(\mathbf{m m})\end{array}$ & $\begin{array}{c}\text { D2 mean } \\
(\mathbf{m m})\end{array}$ & $\begin{array}{c}\text { D3 mean } \\
(\mathbf{m m})\end{array}$ & $\begin{array}{c}\text { D4 mean } \\
(\mathbf{m m})\end{array}$ \\
\hline Male & $7.20 \pm 0.90$ & $4.59 \pm 0.75$ & $6.64 \pm 0.75$ & $0.98 \pm 0.4$ \\
\hline Female & $7.29 \pm 0.96$ & $5.81 \pm 0.85$ & $5.75 \pm 0.69$ & $0.96 \pm 0.3$ \\
\hline p-value & 0.49 & 0.01 & 0.02 & 0.43 \\
\hline \multicolumn{5}{|c|}{ p-value less than 0.05 is taken as significant }
\end{tabular}

Table-3: Mean values of distances measured in male and female

\begin{tabular}{|c|c|c|c|}
\hline $\begin{array}{c}\text { Distance } \\
\text { measured }\end{array}$ & Mean (mm) & $\begin{array}{c}\text { Maximum } \\
(\mathbf{m m})\end{array}$ & $\begin{array}{c}\text { Minimum } \\
(\mathbf{m m})\end{array}$ \\
\hline D5 & $3.77 \pm 0.73$ & 5.71 & 2.4 \\
\hline D6 & $38.22 \pm 2.98$ & 44.35 & 29.18 \\
\hline
\end{tabular}

Table 4: Measurements of trochlear spine and fovea in sagittal plane

Where D5 is the distance measured between fovea/spine and anterior orbital opening

D6 is the distance measured between fovea/spine and optic canal

Like the measurements taken in frontal plane, D5 values also didn't show any statistically significant difference between male $(3.81 \pm 0.77)$ and female $(3.62 \pm 0.73)$. However there was statistically significant difference among values of D6 in male orbits $(38.77 \pm 3.1)$ and female orbits $(35.25 \pm 2.7)$

\section{DISCUSSION}

The surgeries for frontal sinus or even ethmoidal sinus involving an internal approach with a rigid endoscope and external approach via skin crease the upper eyelid, is followed sometimes postoperatively by diplopia. ${ }^{10}$ The diplopia results from damage to the pulley of superior oblique. The anatomical landmark which marks the location of this cartilaginous pulley is the trochlear fovea and spine which in turn is located at the superomedial wall of orbit. ${ }^{11}$ Surgical repair of medial blowout fractures of orbit could also result in damage to the muscle pulley. ${ }^{12}$ Therefore the current study was attempted to document about the incidence, location and variation of fovea and spine in Pakistani population to prevent the unwanted damage to cartilaginous pulley postoperatively.

In this study the incidence of trochlear fovea was found to be $84.17 \%$ while that of trochlear spine was $16.67 \%$. This incidence can be compared with Italian population only since there is no data available on any other population. The frequency of fovea in Italian populace was $73.39 \%$ and that of spine was $15.32 \%{ }^{3}$ Where frequency of fovea was a bit higher in Pakistani population, spine frequency was almost the same. Further it was observed in the study that prevalence of spine was more on the right side than left.

Moreover, it was seen that there was no sex related preference of the occurrence of fovea and spine in female and male orbits. Fovea was seen in $84.2 \%$ of male orbits and $80 \%$ of female orbits. Likewise spine frequency in male $(16.7 \%)$ and female orbits (19.8\%) was not statistically significant. Aglianó M et al (2018) had reported the same finding in their study. 
The position of trochlea was defined by taking two measurements (D5 and D6) in sagittal plane. It was found to be $3.77 \pm 0.73 \mathrm{~mm}$ behind the orbital margin and $38.22 \pm 2.98 \mathrm{~mm}$ in front of optic canal. But the surgeon should keep in mind the variation that it could be found anywhere between 2.4 to 5.7 $\mathrm{mm}$ behind the orbital opening. More explanation on the location of trochlea four measurements (D1, D2, D3 and D4) were taken in frontal plane. In this regard two perpendicular lines were drawn from supra orbital notch and fronto zygomatic suture. The distance of trochlea from supra orbital notch (D1) was found to be $7.22 \pm 0.93 \mathrm{~mm}$ and that from orbital roof (D2) was found to be $4.86 \pm 0.93 \mathrm{~mm}$. Trochlea was found to be at $6.14 \pm 0.83 \mathrm{~mm}$ from fronto zygomatic suture (D3) and at $0.98 \pm 0.48 \mathrm{~mm}$ from medial wall of orbit (D4). The distance (D3) from the horizontal line (L2) ranges between 8.1 and $4.4 \mathrm{~mm}$ while on the contrary the distance (D1) from vertical line (L1) ranges between $8.6 \mathrm{~mm}$ to $5.4 \mathrm{~mm}$.

The measurements which used in this study were quite comparable to the Italian population. ${ }^{3}$ But when we analyzed the measurements in male and female orbits, there was no significant difference statistically in the values of D1, D4 and D5 but D2, D3 and D6 showed significant difference (Table-3). This finding was contrary to the Italian study which reported the significant difference only in the measurement of D6 between male and female orbits. This disparity might be due to the difference in ethnic groups. This difference in D6 between male $(38.77 \pm 3.1)$ and female $(35.25 \pm 2.7)$ should be expected as there is a substantial difference in the average size of male and female orbits. ${ }^{13}$

The present study revealed the precise position of trochlear fovea and spine in Pakistani population. We noticed that the position of fovea from orbital roof and fronto zygomatic suture differed in male and female Pakistani skull, a finding contrary to Italian populace. The reason for this disparity might be race related but still enough data on different ethnic groups is not available. So further research should be done to find out the cause of variation in its location in different populations.

\section{CONCLUSION}

The knowledge of topographic location and variation of trochlear fovea and spine is of extreme significance not only to the ophthalmologists but also to the ENT surgeons for they could prevent postoperative diplopia by sparing the pulley of the superior oblique muscle.

\section{REFERENCES}

1. Haladaj R. Normal anatomy and anomalies of the rectus extraocular muscles in human: a review of the recent data and findings. BioMed Res Int. 2019.

2. Sinnatamby CS. Last's Anatomy regional and applied. 12 ${ }^{\text {th }}$ ed. Edinberg: Elsevier; 2011. Chapter 6, Head and Neck and Spine; p.399-402

3. Aglianó M, Franci D, Volpi N, Orsini D, Messina M, Lorenzoni P. Osteologic topography of the trochlear spine and fovea as landmarks to locate the superior oblique trochlea. Italian Journal of Anatomy and Embryology. 2018; 123:304-11.

4. Standring S. Gray's Anatomy. $41^{\text {st }}$ ed. United Kingdom: Elsevier; 2015. Chapter 41, Orbit and accessory visual apparatus; p. 670-75.

5. Lang J. Clinical Anatomy of Head. Berlin: Springer-Verlag; 2012. Chapter 3, Orbit and Contents; p. 62-3.

6. Locker P, Plitt M, Papagiannopoulos P, Smith $\mathrm{R}$, Tajudeen BA. Anatomic relationship of the first olfactory neuron and trochlea: cadaveric study with surgical implications. Int Forum Allergy Rhinol. 2017; 7:1085-88.

7. Lin JS, Liu TT, Manes RP, Galvin JA. Superior oblique palsy: A complication of endoscopic sinus surgery. JAAPOS. 2015; 19:180-1.

8. Choi J, Lorenz HP, Spain DA. Review of facial trauma management. J Trauma Acute Care Surg. 2020; 88:124-30.

9. Standring S. Gray's Anatomy. 41st ed. United Kingdom: Elsevier; 2015. Chapter 27, External skull; p. 416-28.

10. Beigi B, Vayalambrone D, Kashkouli MB, Prinsley P, Saada J. Combined external and endonasal approach to fronto-ethmoidal mucocele involving the orbit. J Curr ophthalmol. 2016; 28:37-42.

11. Alekseenko S, Karpischenko S. Comparative analysis of the outcome of external and endoscopic frontal sinus surgery in children. Acta Otolaryngol. 2020; 140:687-92.

12. Ji SY, Yoo JH, Ha W, Lee JW, Yang WS. Three cases of acquired simulated brown syndrome after blowout fracture operations. Arch Plast Surg. 2015; 42:346.

13. Avelar LE, Cardoso MA, Bordoni LS, de Miranda Avelar L, de Miranda Avelar JV. Aging and sexual differences of the human skull. Plast Reconstr Surg Glob Open. 2017; 5. 
The Authors:

Dr. Iram Atta

Demonstrator,

Department of Anatomy,

King Edward Medical University, Lahore.

Dr. Maria Nouman

Demonstrator,

Department of Anatomy,

King Edward Medical University, Lahore.
Prof. Raafea Tafweez

Professor and Chairperson,

Anatomy and Histology Department,

King Edward Medical University, Lahore.

\section{Corresponding Author:}

Dr. Maria Nouman

Demonstrator,

Department of Anatomy,

King Edward Medical University, Lahore.

E-mail: marriahnouman@gmail.com 